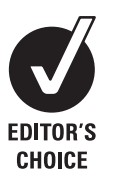

Department of Cardiology, University of Erlangen, Erlangen, Germany

Correspondence to:

Dr M Marwan, Innere Medizin II, Ulmenweg 18, 91054 Erlangen, Germany; mohamed.marwan@ uk-erlangen.de

Accepted 17 November 2008 Published Online First 22 April 2009

\title{
Clinical characteristics of patients with obstructive coronary lesions in the absence of coronary calcification: an evaluation by coronary CT angiography
}

\author{
M Marwan, D Ropers, T Pflederer, W G Daniel, S Achenbach
}

\begin{abstract}
Background: Multidetector CT allows detection of coronary artery calcium and, after contrast injection, visualisation of the coronary artery lumen. It is commonly assumed that the absence of coronary calcification makes the presence of obstructive coronary lesions highly unlikely. This study evaluates the clinical characteristics of patients with at least one symptomatic, high-grade coronary artery stenosis in both computed tomography and invasive angiography but absence of any coronary calcification and compares the results with patients with stenoses in the setting of detectable coronary calcium
\end{abstract}

Patients and methods: The study retrospectively identified 21 consecutive patients with symptoms in whom a high-grade coronary artery stenosis had been identified in 64-slice or dual-source CT coronary angiography (Siemens Sensation 64 or Siemens Definition, $120 \mathrm{kV}, 50$ to $85 \mathrm{ml}$ of intravenous contrast at $5 \mathrm{ml} / \mathrm{s}$ ) in the absence of coronary calcium and in whom that finding had been confirmed by invasive coronary angiography. Clinical presentation ("unstable": all forms of acute coronary syndrome versus "stable": stable chest pain or dyspnoea on exertion) and standard cardiovascular risk factors were assessed, and the results were compared with 42 consecutive patients with symptoms in whom both coronary calcium and coronary stenoses had been identified in computed tomography and invasive coronary angiography.

Results: The majority of patients with coronary stenoses in the absence of coronary calcium presented with "unstable" symptoms (non-ST-segment elevation myocardial infarction (NSTEMI) or unstable angina), significantly more frequently than patients with detectable calcification $(71 \%$ vs $26 \%, p=0.001)$. The age range of patients without calcium was 33 to 76 years, their mean age was younger (53 (SD 13) vs 63 (8) years, $p<0.001$ ), but none of the risk factors showed any significant difference compared with patients with calcification.

Conclusion: The presence of significant coronary artery stenosis in the absence of coronary calcium is possible. It is more likely in the setting of unstable angina or NSTEMI than in stable chest pain and occurs more frequently in younger patients.

The detection of coronary calcifications (coronary calcium score) with electron beam tomography (EBT) and CT has been used for risk stratification. Coronary calcium can be assessed with high sensitivity and quantified by various methods (Agatston score, ${ }^{1}$ volume or mass). The amount of coronary calcium roughly correlates with the amount of coronary atherosclerotic plaque, ${ }^{2}$ and a large number of prospective studies have demonstrated that the absence of coronary calcium clearly indicates a low cardiovascular event risk, whereas increasing amounts of calcium are associated with an increasing event risk..$^{3-8}$ However, there is a striking heterogeneity among human atherosclerotic lesions, and coronary plaques often consist of non-calcified tissue. Thus, even in coronary vessels without calcified plaques, severe atherosclerosis may be present..$^{10}$ All the same, a large number of studies and a total of 7600 patients with symptoms have demonstrated a high sensitivity (95-99\%) of coronary calcium for the presence of coronary artery stenoses along with a high negative predictive value (96-100\%). ${ }^{11}$ However, while the presence of obstructive stenoses in the absence of calcium is unlikely, it is not impossible, and the clinical characteristics of patients with high-grade coronary artery stenoses, but with an absence of detectable coronary calcium, have so far not been analysed.

In this retrospective analysis, we studied the clinical characteristics of patients with obstructive coronary atherosclerotic lesions in the absence of coronary calcifications, and compared them with a consecutive series of patients with obstructive coronary atherosclerotic lesions in the presence of calcium.

\section{PATIENTS AND METHODS}

We retrospectively identified 21 consecutive patients with symptoms who had been evaluated by coronary CT angiography (CTA) during the time period between January 2005 and April 2007-during which a total of about 2300 coronary CTA procedures were performed - and in whom at least one coronary artery stenosis $>70 \%$ diameter reduction had been identified visually by $\mathrm{CT}$, and subsequently confirmed by invasive coronary angiography. We also analysed the patients' baseline clinical characteristics and compared them with 42 consecutive patients with a high-grade stenosis in CT angiography and invasive angiography but in the presence of detectable coronary calcium in CT. The control group, consisting of 42 consecutive patients with stenoses in the presence of calcium, were retrospectively identified over a period of approximately 2 months. All patients were symptomatic and had been referred for CT coronary angiography to exclude significant coronary artery stenosis in the setting of a pretest 
likelihood of disease that was considered "intermediate" based on symptoms, age, gender and the results of tests for ischaemia.

Baseline clinical characteristics included age, sex, body mass index (BMI), hypertension, diabetes mellitus, dyslipidaemia, positive family history for coronary artery disease or stroke and smoking. The clinical presentation was analysed as to whether the patient presented with an acute coronary syndrome (ACS), stable angina or non-anginal pains. Pretest probability for $\mathrm{CAD}$ according to age, gender and symptoms was calculated for both patient groups. ${ }^{12}{ }^{13}$ A patient was considered to have diabetes if they were taking insulin or oral hypoglycaemic drugs or if they had previously received such treatment and were currently controlling the condition through dietary modification. A patient was considered to be hypertensive if they had received such a diagnosis on the basis of arterial blood pressure $>140$ / $90 \mathrm{~mm} \mathrm{Hg}$ or were being treated with antihypertensive medications. A patient was considered to have a positive family history if a first degree relative had history of coronary heart disease or stroke at an age $<65$ years.

Coronary artery calcification scoring and CT angiography were performed using either a 64-slice CT scanner or a dualsource CT scanner (Sensation/Definition, Siemens Health Care, Forchheim, Germany). Scan parameters for calcium screening were $1.2 \mathrm{~mm}$ collimation, $3 \mathrm{~mm}$ slice thickness, $120 \mathrm{kV}$ tube voltage and 165/83 ms temporal resolution for the 64-slice CT/ Dual-source CT scanner, respectively. Scan parameters for CT angiography were $0.6 \mathrm{~mm}$ collimation, $330 \mathrm{~ms}$ rotation time, $120 \mathrm{kV}$ tube voltage and $165 / 83 \mathrm{~ms}$ temporal resolution for the 64-slice CT/Dual-Source CT scanner, respectively. Depending on the scan duration, $50-85 \mathrm{ml}$ of contrast material $(5 \mathrm{ml} / \mathrm{s}$, $350 \mathrm{mg}$ of iodine $/ \mathrm{ml}$ ) was injected, followed by $50 \mathrm{ml}$ of saline chaser. Using a half-scan reconstruction algorithm, overlapping axial cross-sectional images with $0.75 \mathrm{~mm}$ slice thickness and $0.4 \mathrm{~mm}$ increment were reconstructed using a medium sharp convolution kernel (B25f/B26f). For calcium scoring, a standard kernel (B35f) was used for reconstruction of the CT data. Images were retrospectively reconstructed with a slice thickness of $3.0 \mathrm{~mm}$ (increment $3.0 \mathrm{~mm}$ ) for both CT scanners. Calcium scoring was performed on the reconstructed image sets with commercially available software (Syngo CaScore, Siemens, Forchheim, Germany). Three different scoring methods were used: Agatston scoring, volume scoring and equivalent mass scoring. ${ }^{14}$ A standard scoring threshold of $130 \mathrm{HU}$ was used during the procedure.

Calcium was determined to be present if CTA showed a highdensity lesion that could be separated from the contrast enhanced lumen, could be assigned to the coronary wall and could be identified in two independent planes or if the nonenhanced scan visualised calcified plaques with a density of more than 130 Hounsfield units (HU).

Invasive angiography was subsequently performed within a time period of between $2 \mathrm{~h}$ and 2 weeks. A significant stenosis was defined as a stenosis causing a diameter reduction $\geqslant 70 \%$. Stenosis degree was evaluated visually by two experienced observers. All CT and invasive angiography data sets were reviewed after identification of eligible patients based on original reports, and only individuals in whom the presence of stenoses which fulfilled the prespecified criteria were confirmed remained in the analysis.

\section{STATISTICAL METHODS}

A statistical analysis was performed using SPSS for Windows release 15.0 (SPSS, Chicago). All data are expressed as mean (SD) for continuous variables and as percentage ratio for categorical data. Clinical characteristics in the two groups were compared using the Fisher exact test. For continuous variables, the $t$ test was used to detect statistical significance. $p$ Values $<0.05$ were considered significant.

\section{RESULTS}

The mean age of 21 patients with a high-grade stenosis in the absence of coronary calcification was 53 (SD 13) years (range 33-76 years). The mean age of 42 patients with high-grade stenosis and detectable calcium was 63 (8) years (range 4080 years, $p<0.001)$. Of the 21 patients without calcium, 15 $(71 \%)$ presented with unstable symptoms with 13 patients diagnosed as having unstable angina and two patients diagnosed as having non-ST-segment elevation myocardial infarction (NSTEMI), as compared with $11 / 42$ patients $(26 \%)$ in the control group $(p=0.001)$. The 11 patients of the control group included nine patients with unstable angina and two patients with NSTEMI. Other clinical parameters did not show any statistically significant difference between the two groups (see table 1).

The pretest probability for CAD for both patient groups was calculated on the basis of age, gender and symptoms. ${ }^{12}{ }^{13}$ Among the 21 patients with significant stenosis in the absence of any calcification, 16 patients (76\%) had a high pretest probability of $\mathrm{CAD}$, three $(14 \%)$ had an intermediate pretest probability, and two $(10 \%)$ patients had a low pretest probability. Among the 42 patients in the control group, 30 (71\%) patients had a high pretest probability of $\mathrm{CAD}, 11(26 \%)$ had an intermediate pretest probability, and one (3\%) had a low pretest probability. No statistically significant difference was detected as regards the distribution of pretest probability within both patient groups.

Concerning vessel affection, among patients with absent calcification, one patient had a high-grade stenosis of the left main coronary artery, and the remaining 20 had single-vessel disease with 70\% LAD affection (14/20), 20\% LCX affection (4/ $20)$ and $10 \%$ RCA affection (2/20). Assessment of the stenosis degree revealed total occlusion in one patient, and high-grade stenosis in 20. Ninety-three per cent of the 42 patients with coronary calcium suffered single-vessel disesase (39/42)-51\% LAD affection (20/39), 26\% LCX affection (10/39) and 23\% with RCA affection (9/42) —one patient had two-vessel disease (LAD and RCA), and two patients had three-vessel disease. Further assessment of the location of coronary calcification (whether at the site of significant disease or remote from the stenosis) revealed that $93 \%(39 / 42)$ of the patients had calcium at the stenosis, whereas in $7 \%(3 / 42)$ of the patients, the stenosis was caused by non-calcified plaque, and coronary calcium was present in remote vessels (figs 1,2).

\section{DISCUSSION}

Based on investigations which analysed the presence and extent of coronary calcium in patients referred for invasive angiography, the likelihood of obstructive coronary artery lesions in the absence of calcification is considered to be very low. ${ }^{3-8}$ However, the presence of obstructive disease in the absence of coronary calcium is possible. To help characterise the type of patients in whom significant coronary stenoses with absence of calcification may be encountered, we analysed the clinical characteristics of 21 consecutive patients with obstructive $\mathrm{CAD}$ in the absence of coronary calcification as identified by CT and further confirmed by invasive angiography. All patients were symptomatic and had been referred for coronary CT angiography for suspected CAD on the grounds of acute or chronic symptoms. 
Figure 1 Thirty-eight-year-old patient with chronic stable angina. The images show high-grade LCX stenosis caused by non-calcified plaque detected by dualsource CT and validated by invasive coronary angiography. (A) Curved multiplanar reconstruction of the left main and left anterior descending coronary artery. No stenoses were present. (B) Invasive angiogram of the left anterior descending coronary artery. No stenoses were present. (C) Curved multiplanar reconstruction of the left main and left circumflex coronary artery showing a high-grade stenosis in the absence of coronary calcification (arrow). The insert shows a magnified view of the noncalcified plaque causing the high-grade stenosis. (D) Invasive coronary angiogram of the left circumflex coronary artery showing mid-segment high-grade stenosis. (E) Curved multiplanar reconstruction of the right coronary artery showing neither plaques nor stenoses. (F) Invasive coronary angiogram of the right coronary artery. No stenoses were present.
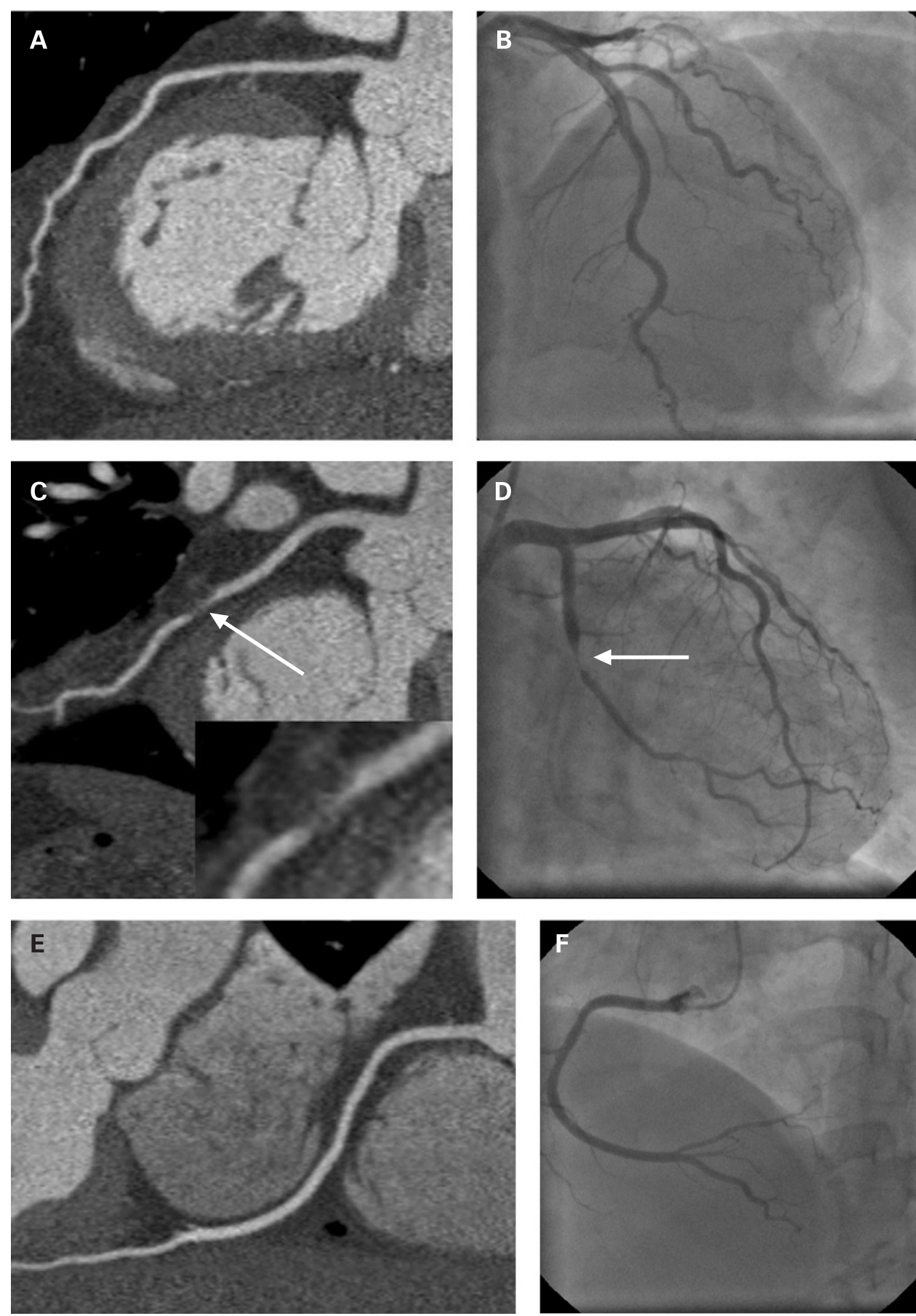

Clinical characteristics were compared with a control group of 42 consecutive patients with high-grade stenoses in the presence of detectable coronary calcification.

We observed that patients with stenoses but with no calcification were younger and more frequently presented with unstable symptoms as compared with patients who had detectable coronary calcium. This is clinically relevant because these are patients who may be more likely to be investigated by coronary CT angiography. CT imaging is recommended in patients with symptoms who are not at a high risk for coronary artery disease and who therefore will typically be younger. Also, the assessment of patients with acute chest pain, absence of ECG changes suggestive of ischaemia and normal myocardial enzymes is among the "appropriate" indications for coronary CTA. ${ }^{15}$

Therefore, the mere absence of calcium, especially in young patients and in patients with acute chest pain referred for CT assessment of possible coronary artery disease, does not rule out the presence of coronary artery stenoses.

However, our study was not designed to predict the likelihood of stenoses in patients without coronary artery calcification, or the percentage of patients without calcification among all those who have coronary artery stenoses. Bamberg et al have reported the presence of exclusively non-calcified plaque to be rare in 195 patients who are investigated by coronary CT angiography. ${ }^{16}$ In a study performed by Cheng et al, the 
Figure 2 Patient with high-grade LAD stenosis in the presence of calcium detected by dual-source CT and validated with invasive coronary angiography. (A) Curved multiplanar reconstruction of the left main and left anterior descending coronary artery showing a high-grade stenosis in the presence of coronary calcification. The insert shows a magnified view of the mixed plaque causing high-grade LAD stenosis. (B) Invasive coronary angiogram of the left anterior descending coronary artery showing a high-grade stenosis in the mid segment (arrow). (C) Curved multiplanar reconstruction of the left main and left circumflex coronary artery. No stenoses were present. (D) Invasive angiogram of the left circumflex coronary artery. No stenoses were present. (E) Curved multiplanar reconstruction of the right coronary artery. No stenoses were present. (F) Invasive coronary angiogram of the right coronary artery. No stenoses were present.
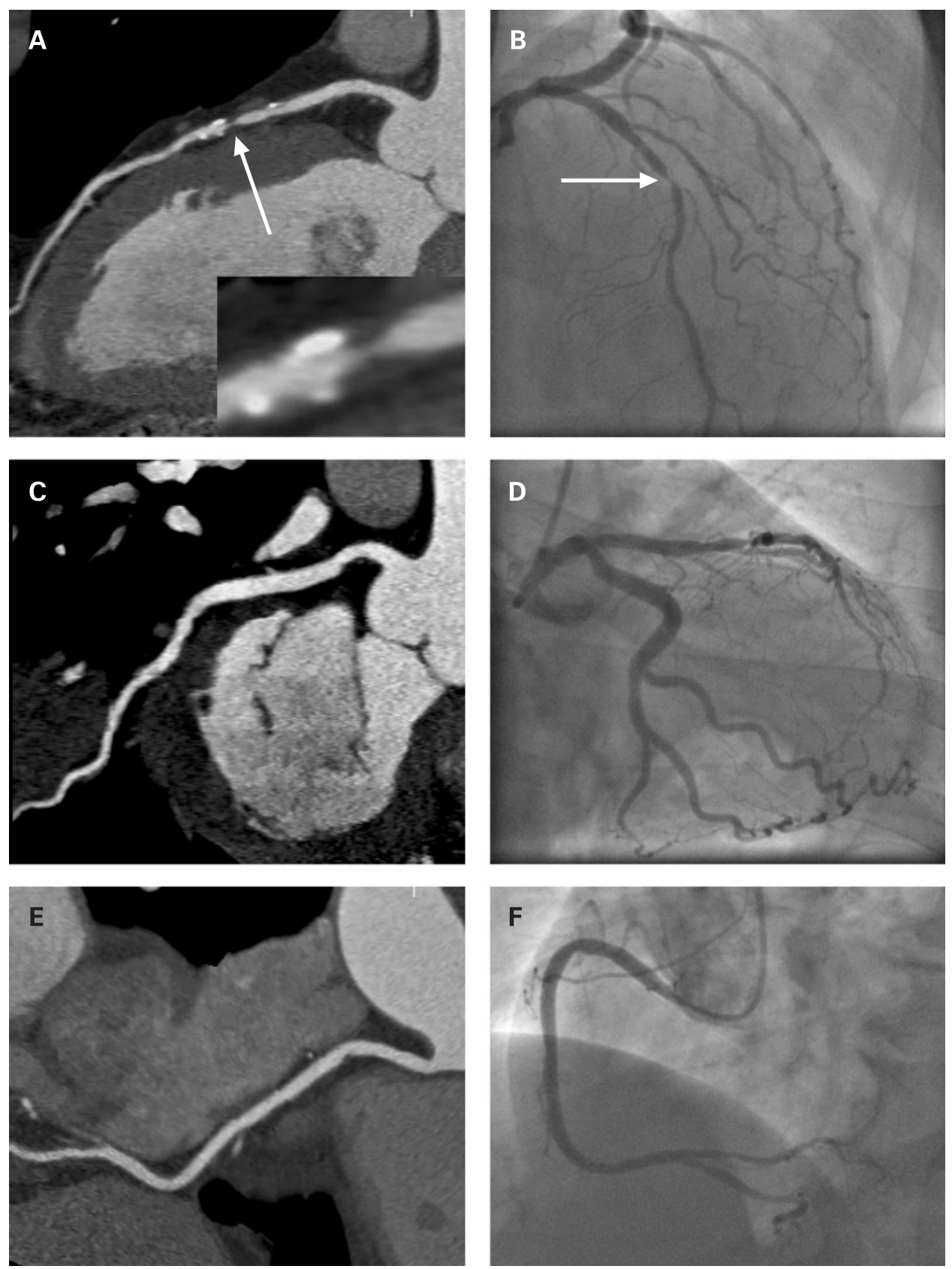

prevalence of non-calcified plaque in 416 patients with a 0 calcium score was $6.5 \%$, with only $0.5 \%$ prevalence of obstructive $\left(>50 \%\right.$ lumen reduction) non-calcified plaque. ${ }^{17}$ On the other hand, Kelly et al recently reported a high prevalence of non-calcified plaque in 325 patients with a calcium score of 0 , with fewer than half of the patients being disease-free and $4 \%$ of the patients showing a stenosis $>50 \%$ on CT angiography. ${ }^{18}$ The authors explain the contradicting

Table 1 Baseline clinical characteristics

\begin{tabular}{llll}
\hline Clinical characteristics & $\begin{array}{l}\text { Patients with detectable coronary } \\
\text { calcification }(\mathbf{n}=\mathbf{4 2})\end{array}$ & $\begin{array}{l}\text { Patients without coronary } \\
\text { calcification }(\mathbf{n}=\mathbf{2 1})\end{array}$ & p Value \\
\hline Age in years (SD), range & $63(8), 40$ to 80 & $53(13), 33$ to 76 & $<0.001$ \\
Unstable presentation & $11(26 \%)$ & $15(71 \%)$ & 0.001 \\
Male gender & $34(81 \%)$ & $16(76 \%)$ & $\mathrm{NS}$ \\
Arterial hypertension & $31(74 \%)$ & $13(62 \%)$ & $\mathrm{NS}$ \\
Diabetes mellitus & $7(17 \%)$ & $1(5 \%)$ & $\mathrm{NS}$ \\
Family history & $14(33 \%)$ & $5(24 \%)$ & $\mathrm{NS}$ \\
Smoking & $19(45 \%)$ & $12(57 \%)$ & $\mathrm{NS}$ \\
BMl & $27.9(5)$ & $26.4(3)$ & $\mathrm{NS}$ \\
\hline
\end{tabular}


findings on the basis of selection bias as well as the presence of a large number of low-risk patients in the Cheng study. They further stress the need for a high suspicion index to detect lowvolume non-calcified plaque. However, two major differences from the data presented in our analysis are that many of the patients in the previous analyses were asymptomatic (53\% in the study by Kelly et al) and that the vast majority did not have significant coronary luminal stenoses. The characteristics of patients with significant stenoses in the absence of calcium have not been previously reported. Our observation that patients with acute symptoms were more frequently encountered among those without calcification is in agreement with previous work showing that lesions associated with acute coronary syndromes are often not calcified. ${ }^{19-22}$

A statistically significant difference could be detected comparing age $(p<0.001)$ and clinical presentation $(p<0.001)$ in the studied groups, with a tendency toward younger age as well as presenting with unstable symptoms in patients with absent coronary calcification.

The relatively small patient group and the retrospective nature of our study are among the limitations. However, these data clearly indicate that coronary stenoses in the complete absence of coronary calcification are most frequently found in patients at a younger age and with unstable chest pain, which may be of relevance for clinical application of cardiac computed tomography. A "negative" calcium score cannot be relied upon, especially in these patient groups, to reliably exclude the presence of significant coronary luminal stenosis.

Funding: This study was supported by a grant from Deutsche Forschungsgemeinschaft, Bonn (AC 91/3-1).

Competing interests: None.

Ethics approval: Ethics approval was provided by University of Erlangen.

\section{REFERENCES}

1. Agatston AS, Janowitz WR, Hildner FJ, et al. Quantification of coronary artery calcium using ultrafast computed tomography. J Am Coll Cardiol 1990:15:827-32.

2. Sangiorgi G, Rumberger JA, Severson A, et al. Arterial calcification and not lumen stenosis is highly correlated with atherosclerotic plaque burden in humans a histologic study of 723 coronary artery segments using nondecalcifying methodology. J Am Coll Cardiol 1998;31:126-33.

3. Shaw LJ, Raggi P, Schisterman E, et al. Prognostic value of cardiac risk factors and coronary artery calcium screening for all-cause mortality. Radiology 2003;228:82633.

4. Raggi P, Callister TQ, Cooil N, et al. Identification of patients at increased risk of first unheralded acute myocardial infarction by electron-beam computed tomography. Circulation 2000;101:850-5
5. Vliegenthart R, Oudkerk M, Hofman A, et al. Coronary calcification improves cardiovascular risk prediction in the elderly. Circulation 2005;112:572-7.

6. Greenland $\mathbf{P}$, LaBree L, Azen SP, et al. Coronary artery calcium score combined with Framingham score for risk prediction in asymptomatic individuals. JAMA 2004;291:210-15.

7. Arad Y, Goodman KJ, Roth $\mathrm{M}$, et al. Coronary calcification, coronary disease risk factors, C-reactive protein, and atherosclerotic cardiovascular disease events the St. Francis Heart Study. J Am Coll Cardiol 2005;46:158-65.

8. Brown ER, Kronmal RA, Bluemke DA, et al. Coronary calcium coverage score: determination, correlates, and predictive accuracy in the multi-ethnic study of atherosclerosis. Radiology 2008;247:669-75.

9. Virmani R, Kolodgie F, Burke A, et al. Lessons from sudden coronary death: A comprehensive morphological classification scheme for atherosclerotic lesions. Arterioscler Thromb Vasc Biol 2000;20:1262-75.

10. Kragel A, Reddy S, Wittes J, et al. Morphometric analysis of the composition of atherosclerotic plaques in the four major epicardial coronary arteries in acute myocardial infarction and in sudden coronary death. Circulation 1989;80:1747-56.

11. Budoff MJ, Achenbach S, et al. Assessment of Coronary artery disease by cardiac computed tomography: a scientific statement From the American Heart Association Committee on Cardiovascular Imaging and Intervention, Council on Cardiovascular Radiology and Intervention, and Committee on Cardiac Imaging, Council on Clinical Cardiology. Circulation 2006;114:1761-91.

12. Diamond GA, Forrester JS. Analysis of probability as an aid in the clinical diagnosis of coronary-artery disease. New Engl J Med 1979;300:1350-8.

13. Weiner DA, Ryan TJ, McCabe $\mathrm{CH}$, et al. Exercise stress testing. Correlations among history of angina, ST-segment response and prevalence of coronary-artery disease in the Coronary Artery Surgery Study (CASS). New Engl J Med 1979;301:230-5.

14. Becker CR, Knez A, Ohnesorge B, et al. Visualization and quantification of coronary calcifications with electron beam and spiral computed tomography. Eur Radiol 2000;10:629-35.

15. Hendel R, Patel M, Kramer C, et al. ACCF/ACR/SCCT/SCMR/ASNC/NASCI/SCAI/SIR 2006 appropriateness criteria for cardiac computed tomography and cardiac magnetic resonance imaging. J Am Coll Cardiol 2006;48:1475-97.

16. Bamberg F, Dannemann N, Shapiro MD, et al. Association between cardiovascular risk profiles and the presence and extent of different types of coronary atherosclerotic plaque as detected by multidetector computed tomography. Arterioscler Thromb Vasc Biol 2008;28:568-74.

17. Cheng VY, Lepor NE, Madyoon $\mathrm{H}$, et al. Presence and severity of noncalcified coronary plaque on 64-slice computed tomographic coronary angiography in patients with zero and low coronary artery calcium. Am J Cardiol 2007;99:1183-6.

18. Kelly JL, Thickman D, Abramson SD, et al. Coronary CT angiography findings in patients without coronary calcification. AJR Am J Roentgenol 2008;191:50-5.

19. Hoffmann U, Moselewski F, Nieman K, et al. Noninvasive assessment of plaque morphology and composition in culprit and stable lesions in acute coronary syndrome and stable lesions in stable angina by multidetector computed tomography. J Am Coll Cardiol 2006;47:1655-62.

20. Leber AW, von Ziegler F, Becker A, et al. Characteristics of coronary plaques before angiographic progression determined by multi-slice CT. Int J Cardiovasc Imaging 2008:24:423-8.

21. Schuijf JD, Beck T, Burgstahler C, et al. Differences in plaque composition and distribution in stable coronary artery disease versus acute coronary syndromes; noninvasive evaluation with multi-slice computed tomography. Acute Card Care 2007;9:48-53.

22. Motoyama S, Kondo T, Sarai M, et al. Multislice computed tomographic characteristics of coronary lesions in acute coronary syndromes. J Am Coll Cardiol 2007:50:319-26. 